\title{
Evaluation of easily measured risk factors in the prediction of osteoporotic fractures
}

\author{
Robert Bensen*1, Jonathan D Adachi², Alexandra Papaioannou ${ }^{2}$, \\ George Ioannidis ${ }^{1}$, Wojciech P Olszynski ${ }^{3}$, Rolf J Sebaldt ${ }^{2}$, \\ Timothy M Murray ${ }^{4}$, Robert G Josse ${ }^{4}$, Jacques P Brown ${ }^{5}$, David A Hanley ${ }^{6}$, \\ Annie Petrie ${ }^{1}$, Mark Puglia ${ }^{2}$, Charlie H Goldsmith ${ }^{1}$ and W Bensen ${ }^{2}$
}

\author{
Address: ${ }^{1}$ Medical Science, McMaster University, Hamilton, Ontario, Canada, ${ }^{2}$ Medicine, McMaster University, Hamilton, Ontario, Canada, \\ ${ }^{3}$ Medicine, University of Saskatchewan, Saskatoon, Saskatchewan, Canada, ${ }^{4}$ Medicine, University of Toronto, Toronto, Ontario, Canada, \\ ${ }^{5}$ Medicine, Laval University, Ste-Foy, Quebec, Canada and ${ }^{6}$ Medicine, University of Calgary, Calgary, Alberta, Canada \\ Email: Robert Bensen* - bensenrw@gmail.com; Jonathan D Adachi - jd.adachi@sympatico.ca; Alexandra Papaioannou - papaioannou@hhsc.ca; \\ George Ioannidis - g.ioannidis@sympatico.ca; Wojciech P Olszynski - olszynski@webster.sk.ca; Rolf J Sebaldt - sebaldt@mcmaster.ca; \\ Timothy M Murray - tim.murray@utoronto.on.ca; Robert G Josse - josser@smh.utoronto.on.ca; \\ Jacques P Brown - jacques.brown@crchul.ulaval.ca; David A Hanley - dahanley@ucalgary.ca; Annie Petrie - petriea@mcmaster.ca; \\ Mark Puglia - pugliam@mcmaster.ca; Charlie H Goldsmith - goldsmith@mcmaster.ca; W Bensen - wbensen@cogeco.ca \\ * Corresponding author
}

Published: 05 September 2005

BMC Musculoskeletal Disorders 2005, 6:47 doi:10.1 I86/147/-2474-6-47

This article is available from: http://www.biomedcentral.com/I47/-2474/6/47

(C) 2005 Bensen et al; licensee BioMed Central Ltd.

This is an Open Access article distributed under the terms of the Creative Commons Attribution License (http://creativecommons.org/licenses/by/2.0), which permits unrestricted use, distribution, and reproduction in any medium, provided the original work is properly cited.

\begin{abstract}
Background: Fracture represents the single most important clinical event in patients with osteoporosis, yet remains under-predicted. As few premonitory symptoms for fracture exist, it is of critical importance that physicians effectively and efficiently identify individuals at increased fracture risk.

Methods: Of 3426 postmenopausal women in CANDOO, 40, 158, 99, and 64 women developed a new hip, vertebral, wrist or rib fracture, respectively. Seven easily measured risk factors predictive of fracture in research trials were examined in clinical practice including: age $(<65,65-69,70-74,75-79,80+$ years $)$, rising from a chair with arms (yes, no), weight $(<57, \geq \mathbf{5 7} \mathrm{kg}$ ), maternal history of hip facture (yes, no), prior fracture after age 50 (yes, no), hip T-score (>-I, -I to >-2.5, $\leq-2.5$ ), and current smoking status (yes, no). Multivariable logistic regression analysis was conducted.

Results: The inability to rise from a chair without the use of arms $(3.58 ; 95 \% \mathrm{Cl}$ : I. 17, 10.93) was the most significant risk factor for new hip fracture. Notable risk factors for predicting new vertebral fractures were: low body weight (1.57; 95\% Cl: I.04, 2.37), current smoking (I.95; 95\% Cl: $1.20,3.18$ ) and age between $75-79$ years (I.96; 95\% Cl: I. I0, 3.5I). New wrist fractures were significantly identified by low body weight (I.7I, $95 \% \mathrm{Cl}:$ I.0I, $2.90)$ and prior fracture after 50 years $(1.96 ; 95 \% \mathrm{Cl}: \mathrm{I} .19,3.22)$. Predictors of new rib fractures include a maternal history of a hip facture $(2.89 ; 95 \% \mathrm{Cl}: 1.04,8.08)$ and a prior fracture after 50 years $(2.16 ; 95 \% \mathrm{Cl}: 1.20,3.87)$.

Conclusion: This study has shown that there exists a variety of predictors of future fracture, besides BMD, that can be easily assessed by a physician. The significance of each variable depends on the site of incident fracture. Of greatest interest is that an inability to rise from a chair is perhaps the most readily identifiable significant risk factor for hip fracture and can be easily incorporated into routine clinical practice.
\end{abstract}




\section{Background}

Osteoporosis affects one in four women and one in eight men in Canada [1]. Its consequences are devastating both to the individual and society in terms of suffering, disability, and increased health care expenses. It is expected that over the next 40 years the number of hip fractures alone will increase exponentially, leading to a problem of epidemic proportions [2]. Fragility fractures are the most important and disabling consequence of osteoporosis and result in a loss of functional ability and increased morbidity and mortality. Estimated conservatively, a 50 year old woman has a 40 percent lifetime risk of a hip, vertebral or wrist fracture [3]. In 1993, the Canadian health care system spent more than $\$ 1.3$ billion on osteoporosis-related fractures [4]. Recent research has shown that the mean one-year cost of a hip fracture, including direct and indirect costs, is (CAN) \$26 527 [5].

Predicting incident fractures is critical today, but in light of the aging demographics, will have even greater importance in the future. Early recognition and treatment of osteoporotic patients is crucial to the prevention of these fractures. A number of risk factors have come to define those at increased risk for osteoporotic fracture and much work has been done in an attempt to delineate the critical variables [6-11]. In order for risk assessment to be effective and efficient, it must be practical and have high predictive value for the identification of fractures. Black et al., with use of data from the Study of Osteoporotic Fractures (SOF), developed the Fracture Index [12]. This assessment tool for predicting fracture risk in osteoporotic post-menopausal women was shown to be predictive of incident osteoporotic fractures (hip, vertebral, wrist and rib) in post-menopausal women and was validated using the EPIDOS study [12]. The Fracture Index assessment tool considers seven variables: age, fracture after age 50, history of maternal hip fracture after age 50, weight less than $125 \mathrm{lb}$ ( $57 \mathrm{~kg}$ ), smoking status, use of arms to stand up from a chair and bone mineral density (BMD) T-score [12]. These variables were chosen not only based on their predictive value but also because of their ease of assessment in a clinical setting. As a result, we sought to examine the usefulness of these risk factors in a clinical specialist setting.

The purpose of this study was to examine whether the risk factors outlined in the Fracture Index could be used to predict new incident osteoporotic fractures of the hip, vertebra, wrist, and rib in postmenopausal women who are registered in The Canadian Database for Osteoporosis and Osteopenia (CANDOO).

\section{Methods \\ Study design}

An analysis using prospective collected patient information from the CANDOO database was conducted. The CANDOO registry is a multi-site (Calgary, Winnipeg, Saskatoon, Toronto, Hamilton, Montreal, Quebec City) database consisting of more than 10,000 men and women who have been referred to specialists for osteoporosis [13]. The CANDOO database is designed to gather osteoporosis-related clinical information in a prospective manner displaying a record at each clinical visit. Patient information is entered and recorded into a central database [13]. It includes information on patient demographics; vertebral, rib, wrist and hip fracture history; gynecological history; use of osteoporosis related drugs; drug side effects; use of corticosteroids and other medications; dietary calcium intake; smoking habits; physical activity; fall history; prior medical history; family history including fractures; a self administered osteoporosis health related quality of life instrument; basic laboratory results and bone density measurements [13]. Patients were followed yearly with detailed assessments at clinical centres. Each yearly visit involved the collection of information similar to that collected at baseline.

All centres with a baseline CANDOO assessment (visit 1) between 1990 and 1999 with follow-up data available were selected. Only postmenopausal women $\geq 55$ years of age with follow-up data available entered in CANDOO were considered for inclusion. Individuals with no followup assessments or males were excluded. Any individuals experiencing multiple fractures were also excluded in order to group subjects into hip, vertebral, wrist or rib fracture groups respectively. This exclusion criteria limited the number of applicable subjects from CANDOO and simultaneously implicated only females in the analysis. Fractures in CANDOO were determined based on either self-reports or X-ray confirmation.

For the current study we examined the seven easily measured clinical risk factors for fracture outlined in the Fracture Index. Each risk factor was expressed as an independent variable. The major dichotomous risk factors abstracted were: vertebral, rib, wrist or hip fractures after age 50 (yes/no); maternal family history of fracture (yes/ no); weight $<57 \mathrm{~kg}$ ( $\leq 57 \mathrm{~kg}$, > $57 \mathrm{~kg})$; smoking status (yes/no); use of arms to stand from seated position (yes/ no). Non-dichotomous risk factors abstracted included: age $(<65,65-69,70-74,75-79,80-84,85+$ years $)$; bone mineral density total hip T-score $(>-1,-1$ to $-2.5, \leq-2.5)$ (measurements were made using dual energy X-ray absorptiometry using Lunar or Hologic densitometers) [13]. Adjustments for osteoporotic drug treatment and inclusion of the osteoporosis quality of life questionnaire (OQLQ) as a variable were considered. 
Table I: Baseline characteristics of risk factors for patient cohort.

\begin{tabular}{lc}
\hline RISK FACTOR & n (\% of cohort) \\
\hline Age $<64.5$ years & $1259(36.8)$ \\
Age 64.5-69.5 & $740(21.6)$ \\
Age 69.5-74.5 & $751(21.9)$ \\
Age 74.5-79.5 & $426(12.4)$ \\
Age 79.5-84.5 & $186(5.4)$ \\
Age $\geq 84.5$ & $64(1.9)$ \\
Total Hip T-score, $T>-1$ & $89(19.9)$ \\
Total Hip T-score, $-2.5<T<-1$ & $245(54.8)$ \\
Total Hip T-score, T $\leq-2.5$ & $113(25.3)$ \\
Maternal history of hip fracture & $192(5.6)$ \\
Prior fracture $>50$ years & $1413(41.2)$ \\
Current smokers & $361(10.5)$ \\
Rise from a chair with arms & $1242(49.2)$ \\
Weight $<57$ kg & $867(30.2)$
\end{tabular}

\section{Statistical analysis}

Using the seven independent variables we conducted multivariate logistic regression analyses for each fracture type to identify which of the seven risk factors were related to fracture in patients from the CANDOO database.

While longitudinal databases are a powerful research tool, a common weakness is that of missing or incomplete data. To minimize the bias associated with missing data, multiple imputation was utilized to replace missing data prior to the analyses $[14,15]$. In this case, 10 complete data sets were generated. The multiple imputed datasets were then analyzed using standard procedures for complete data and the results were pooled. Odds ratios and the associated $95 \%$ confidence intervals were calculated.

\section{Results}

\section{Baseline characteristics}

A total of 3426 patients were evaluated. Of those patients examined, 99 developed a wrist fracture, 64 a rib fracture, 158 a vertebral fracture, 40 a new hip fracture and 3065 experienced no fracture. Individuals in CANDOO who developed multiple fractures, 53 in total, were excluded from the analyses. The mean age and weight of the patients was 68 years and $64 \mathrm{~kg}$, respectively. Mean total hip T-score was -1.9.

Baseline characteristics for the risk factors examined are displayed in Table 1 . More than one third $(36.8 \%)$ of the women included were $<65$ years of age. While many subjects did not have a BMD assessment, $54.8 \%$ of those that did had a T-score between -1 and -2.5 . Only $5.6 \%$ of postmenopausal women in CANDOO reported having a maternal history of hip fracture while $41.2 \%$ had a prior fracture after the age of 50 years and $10.5 \%$ were current smokers. Of the entire group, approximately half were only able to rise from a chair with the use of arms (as compared with no arms) and $30.2 \%$ of patients weighed less the $57 \mathrm{~kg}$.

\section{Hip fractures}

The results of multivariable logistic regression analyses for each of the seven risk factors examined to predict a new fracture event in the hip are presented in Table 2. Prior fracture after the age of 50 years, those currently smoking, those between 75-79 years of age and those with a T-score of $<-2.5$ indicated a trend towards higher risk of developing a new hip fracture. The one factor that was statistically significant for predicting subsequent hip fractures were those individuals only able to rise from a chair with the aid of their arms. These individuals had an approximate odds ratio of 3.6. The $95 \%$ confidence interval of each risk factor predicting higher risk of developing a new fracture at the hip site is shown in Figure 1. All the factors had fairly wide confidence intervals, in part due to the overall low incidence of hip fractures. Maternal history of hip fracture was excluded from this model as the hip fracture group contained no data on maternal history of hip fracture.

\section{Vertebral fractures}

The results of multivariate logistic regression analyses for each of the seven risk factors thought to predict a new vertebral fracture within the CANDOO database are depicted in Figure 2 and Table 3. Of all the risk factors, three were found to be significant predictors of new spinal fractures. Current smokers and those between the ages of 75-79 years were approximately two times more likely to develop a new vertebral fracture. Individuals weighing less than $57 \mathrm{~kg}$ were about 1.6 times more likely to develop a new fracture at this site. The other four risk factors did not reach statistical significance in terms of their predictability of a new fracture at vertebral sites in CANDOO.

\section{Wrist fractures}

Table 4 shows odds ratios and $95 \%$ confidence intervals for each risk factor for fractures at the wrist. Each of the seven risk factors' independent ability to predict a new wrist fracture is illustrated in Figure 3. Prior fracture after the age of 50 and a body weight less than $57 \mathrm{~kg}$ were deemed significant risk factors for a new incident wrist fracture. Individuals with a prior fracture after the age of 50 years and those weighing less than $57 \mathrm{~kg}$ were about two and 1.7 times more likely to develop a new wrist fracture, respectively. The other five risk factors were not statistically significant.

\section{Rib fractures}

Individuals with a maternal history of a hip fracture and a prior fracture after the age of 50 years were approximately 
Table 2: Hip Fracture Results Odds ratios and $95 \%$ confidence intervals point estimates for each risk factor in the development of new hip fracture

\begin{tabular}{|c|c|c|}
\hline RISK FACTOR & Odds Ratio & 95\% Confidence Interval \\
\hline Weight $(<57 \mathrm{Kg}) *$ Reference Level: $\geq 57 \mathrm{Kg}$ & 1.70 & $0.719 ; 4.037$ \\
\hline Use of Arms to Rise *Reference Level: rise without arms & 3.58 & $1.173 ; 10.927$ \\
\hline Smoking Status *Reference Level: no smoking & 1.48 & $0.485 ; 4.536$ \\
\hline Age $(65$ to 69 y) $*$ Reference Level: $<65$ & 1.53 & $0.505 ; 4.667$ \\
\hline Age $(70-74$ y) *Reference Level: $<65$ & 0.98 & $0.280 ; 3.421$ \\
\hline Age $(75-79$ y) *Reference Level: $<65$ & 1.62 & $0.45 \mathrm{I} ; 5.840$ \\
\hline Age $(80-84$ y) *Reference Level: $<65$ & 1.52 & $0.296 ; 7.780$ \\
\hline Age $(85+y) *$ Reference Level: $<65$ & 4.36 & $0.823 ; 23.078$ \\
\hline $\begin{array}{l}\text { Previous Fracture After } 50 \text { y } \\
\text { *Reference Level: no fracture after } 50\end{array}$ & 1.08 & $0.477 ; 2.463$ \\
\hline $\begin{array}{l}\text { Osteoporotic (<-2.5 SD)** } \\
\text { *Reference Level: normal BMD }\end{array}$ & 1.69 & $0.233 ; 12.253$ \\
\hline
\end{tabular}

RESULTS: HIP

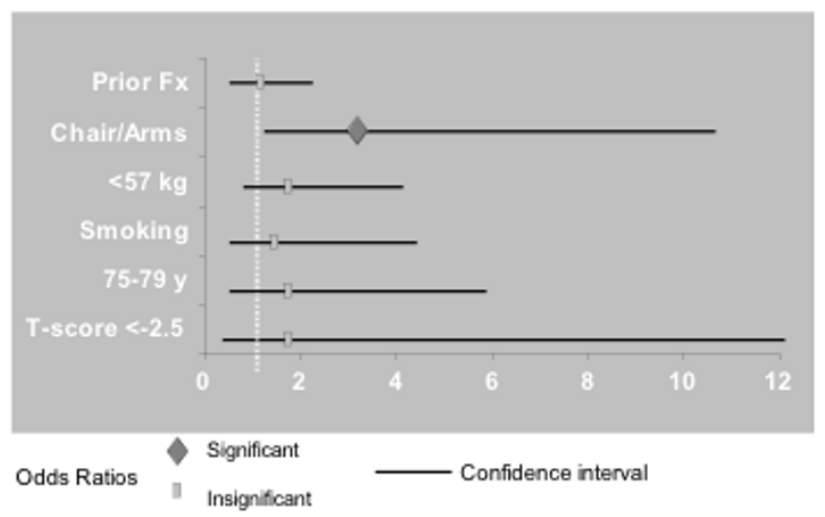

Figure I

Odds ratios and confidence intervals for each risk factor that predicts higher risk of hip fracture.

three and two times more likely, respectively, to develop a new rib fracture during the course of the study. Those with any of the other five risk factors were not at significantly increased risk of sustaining a new fracture at the rib. These data are displayed in Table 5 and Figure 4.

\section{Discussion}

Even with the advent of the recent publication of the 2002 Osteoporosis Society of Canada's Clinical Practice Guidelines, there remains no universal way of identifying those with osteoporosis and those at increased risk of fracture. The clinical goal must be to identify the substantial percentage of the population at high risk for fracture while simultaneously limiting any unnecessary testing to the

\section{RESULTS: SPINE}

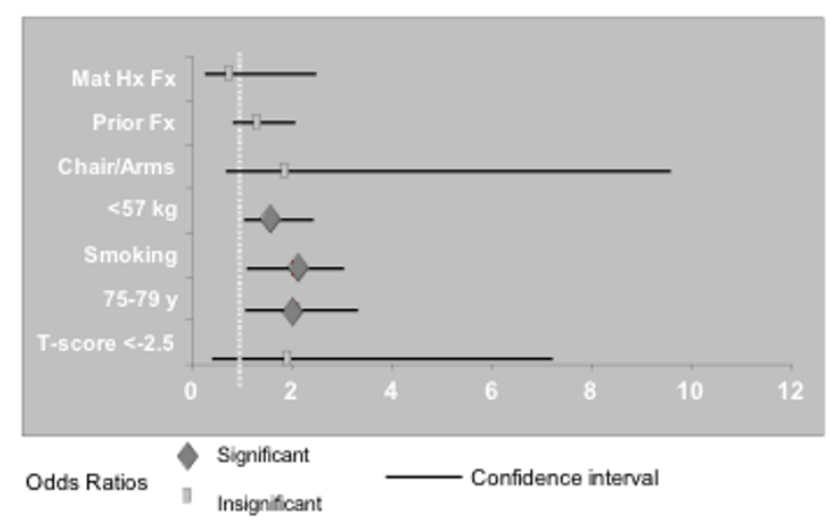

Figure 2

Odds ratios and confidence intervals for each risk factor that predicts higher risk of vertebral fracture.

increasingly burdened health care system $[16,17]$. Prevention of an incident fracture and the cascade of subsequent fractures is the ultimate objective.

We found a variety of clinical risk factors in conjunction with BMD to be extremely helpful in distinguishing those at high risk of fracture in CANDOO.

The association of increasing age with declining bone density has long been recognized as being the predominant risk factor for fracture. Age was shown to be an independent predictor of hip fracture in the EPIDOS study [6]. For ages 45 through 85 , the ten year probability of a fracture in the forearm, humerus, spine or hip increases five 
Table 3: Vertebral Fracture Results Odds ratios and $95 \%$ confidence intervals point estimates for each risk factor in the development of a new vertebral fracture

\begin{tabular}{|c|c|c|}
\hline RISK FACTOR & Odds Ratio & 95\% Confidence Interval \\
\hline Weight $(<57 \mathrm{Kg}) *$ Reference Level: $\geq 57 \mathrm{Kg}$ & 1.57 & $1.035 ; 2.373$ \\
\hline Use of Arms to Rise *Reference Level: rise without arms & 1.72 & $0.981 ; 3.023$ \\
\hline Smoking Status *Reference Level: no smoking & 1.95 & $1.199 ; 3.184$ \\
\hline Age $(65$ to 69 y) $*$ Reference Level: $<65$ & 1.36 & $0.805 ; 2.309$ \\
\hline Age $(70-74$ y) *Reference Level: $<65$ & 1.28 & $0.753 ; 2.174$ \\
\hline Age $(75-79$ y) *Reference Level: $<65$ & 1.96 & $1.096 ; 3.508$ \\
\hline Age $(80-84$ y) *Reference Level: $<65$ & 0.93 & $0.368 ; 2.334$ \\
\hline Age $(85+y) *$ Reference Level: $<65$ & 1.32 & $0.375 ; 4.631$ \\
\hline $\begin{array}{l}\text { Previous Fracture After } 50 \text { y } \\
\text { *Reference Level: no fracture after } 50\end{array}$ & 1.37 & $0.931 ; 2.012$ \\
\hline $\begin{array}{l}\text { Maternal History of Fracture } \\
\text { *Reference Level: no family history }\end{array}$ & 0.85 & $0.296 ; 2.412$ \\
\hline $\begin{array}{l}\text { Osteoporotic (<-2.5 SD)** } \\
\text { *Reference Level: normal BMD }\end{array}$ & 1.85 & $0.448 ; 7.676$ \\
\hline
\end{tabular}

\section{RESULTS: WRIST}

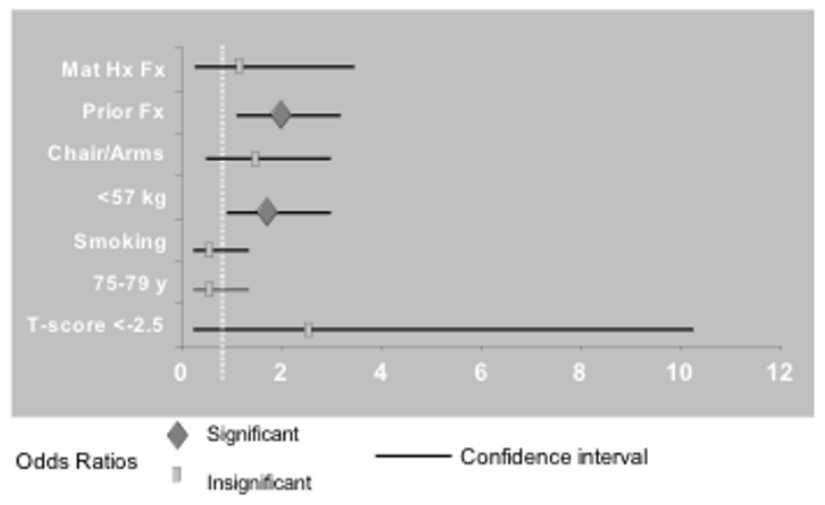

Figure 3

Odds ratios and confidence intervals for each risk factor that predicts higher risk of wrist fracture.

times in men and eight times in women [18]. It has been estimated by Kanis et al. that risk of fracture in the forearm alone can increase eight times between the ages of 45 and 85 [18]. The results from the CANDOO database showed that although age, especially between 75 to 79 years, was associated with heightened risk of fracture at all sites, it only reached significance for predicting future fractures at vertebral sites. These results indicate that it is possible that age alone may not be definitive in predicting overall fracture risk.
In a clinical setting, BMD remains the gold-standard in assessing fracture risk so long as it is considered within the context of age. Cummings et al. showed that for each standard deviation decline in femoral neck BMD is associated with 2.6 times the risk of hip fracture in postmenopausal women aged 65 years or more [19]. Although BMD can identify people who are at increased risk of experiencing a fracture, it cannot, with any certainty, identify those individuals who will necessarily sustain a future fracture. Moreover, BMD testing is both inconvenient and expensive. Many under-serviced areas do not have the technology available for assessing BMD and even in those areas where machines are available, such procedures may be difficult to access due to mobility issues for osteoporotic patients and elderly subjects in long-term care facilities. Results from the CANDOO database did not indicate the overwhelming importance of BMD values in predicting those at increased fracture risk at any of the four sites considered. While a clinically relevant association was seen between BMD and fracture, its association was not statistically significant. This could at least in part arise from the small number of eligible subjects in CANDOO with BMD assessments.

Previous fracture history is well known to be predictive of future fracture risk $[10,20-23]$. The number of prior fractures at the site of incident fracture (i.e. hip, spine) combined with age has been shown to increase fracture risk 1.5 to 9.5 times $[10,20,21,23-27]$. Prior fracture at vertebral sites increases the risk of future fracture by as much as four times $[28,29]$. A previous history of fracture represented an important factor in evaluating the risk of future non-vertebral (wrist and rib) fractures in the CANDOO patients. At hip related sites within the CANDOO population, previous fracture history was not significant in 
Table 4: Wrist Fracture Results Odds ratios and $95 \%$ confidence intervals point estimates for each risk factor in the development of a new fracture at the wrist

\begin{tabular}{|c|c|c|}
\hline RISK FACTOR & Odds Ratio & 95\% Confidence Interval \\
\hline Weight $(<57 \mathrm{Kg}) *$ Reference Level: $\geq 57 \mathrm{Kg}$ & 1.71 & $1.007 ; 2.897$ \\
\hline Use of Arms to Rise *Reference Level: rise without arms & 1.53 & $0.790 ; 2.981$ \\
\hline Smoking Status *Reference Level: no smoking & 0.62 & $0.259 ; 1.466$ \\
\hline Age $(65$ to 69 y) *Reference Level: $<65$ & 0.76 & $0.387 ; 1.485$ \\
\hline Age $(70-74$ y) *Reference Level: $<65$ & 1.00 & $0.539 ; 1.843$ \\
\hline Age $(75-79$ y) *Reference Level: $<65$ & 0.57 & $0.238 ; 1.387$ \\
\hline Age $(80-84$ y) *Reference Level: $<65$ & 0.46 & $0.134 ; 1.603$ \\
\hline Age $(85+y) *$ Reference Level: $<65$ & 0.83 & $0.183 ; 3.776$ \\
\hline $\begin{array}{l}\text { Previous Fracture After } 50 \text { y } \\
\text { *Reference Level: no fracture after } 50\end{array}$ & 1.96 & $1.191 ; 3.223$ \\
\hline $\begin{array}{l}\text { Maternal History of Fracture } \\
\text { *Reference Level: no family history }\end{array}$ & 1.25 & $0.430 ; 3.627$ \\
\hline $\begin{array}{l}\text { Osteoporotic (<-2.5 SD)** } \\
\text { *Reference Level: normal BMD }\end{array}$ & 2.26 & $0.476 ; 10.682$ \\
\hline
\end{tabular}

\section{RESULTS: RIBS}

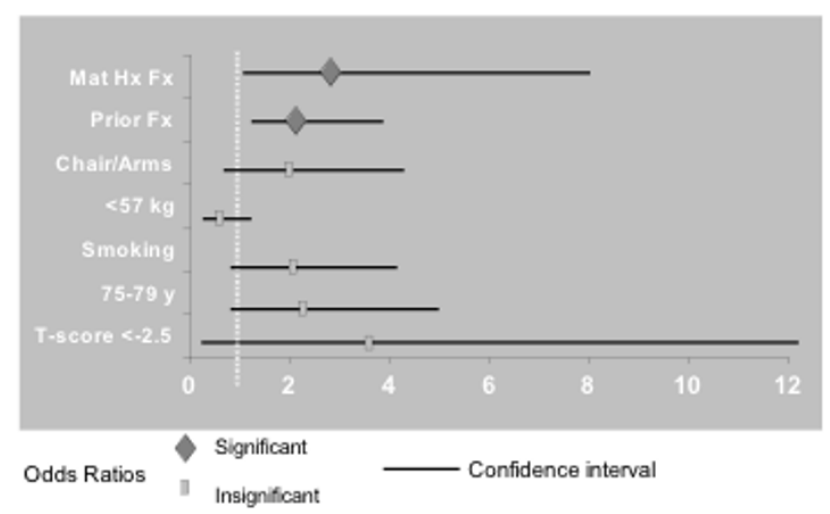

Figure 4

Odds ratios and confidence intervals for each risk factor that predicts higher risk rib fracture.

predicting future hip fracture risk. This may be explained by the low incidence of hip fractures and the fact that many vertebral fractures remain undiagnosed. In fact, it has been estimated that less than one third of all vertebral fractures are clinically diagnosed [30].

Much evidence has indicated that those with a maternal history of fracture, especially at the hip, are at increased risk of future fracture. Moreover, those whose maternal history involves a grandmother, carry an even greater risk of hip fracture [11]. Within the CANDOO patients, the predictive ability of this variable appeared to be less important. At all sites, with the exception of the rib, maternal family history of fracture showed no significant association with increased fracture risk. This could possibly be explained by poor recollection or documentation of maternal history within the baseline assessment.

Low body weight was shown to be a significant predictor of future fracture at both vertebral and wrist sites. It played a significant role in assessing fracture risk at these sites, but appeared to be less important in determining risk of future fracture in the hip or rib.

The direct effects of smoking resulting in declining BMD and increased fracture risk have been identified by a variety of different studies [31-34]. Individuals who reported smoking in CANDOO were only at significantly increased risk of fracture at vertebral sites. These results indicate that at least in CANDOO, smoking as a risk factor is limited at other sites.

Many risk factors incorporated into the Fracture Index and other publications focus on biological, historical or BMDaffecting variables in risk factor analysis. Although the importance of these particular risk factors is unquestionable, a considerable number of osteoporotic fractures result from falls. Easily assessed neuromuscular measures of fallrelated hip fracture have been examined in a few studies $[10,35,36]$. With the use of data from EPIDOS, DargentMolina et al. found that four predictors of fall-related fracture (slow gait speed, difficulty in tandem walking, reduced visual acuity, small calf circumference) were significantly associated with increased risk of future hip fracture [35]. A similar study confirmed that a simple and 
Table 5: Rib Fracture Results Odds ratios and $95 \%$ confidence intervals point estimates for each risk factor in the development of a new fracture at the rib

\begin{tabular}{|c|c|c|}
\hline RISK FACTOR & Odds Ratio & 95\% Confidence Interval \\
\hline Weight $(<57 \mathrm{Kg}) *$ Reference Level: $\geq 57 \mathrm{Kg}$ & 0.47 & $0.222 ; 1.011$ \\
\hline Use of Arms to Rise *Reference Level: rise without arms & 1.93 & $0.868 ; 4.272$ \\
\hline Smoking Status *Reference Level: no smoking & 1.94 & $0.903 ; 4.179$ \\
\hline Age $(65$ to 69 y) $*$ Reference Level: $<65$ & 1.42 & $0.631 ; 3.210$ \\
\hline Age $(70-74$ y) *Reference Level: $<65$ & 1.58 & $0.709 ; 3.516$ \\
\hline Age $(75-79$ y) *Reference Level: $<65$ & 2.26 & $0.928 ; 5.486$ \\
\hline Age $(80-84$ y) *Reference Level: $<65$ & 2.69 & $0.950 ; 7.625$ \\
\hline Age $(85+y) *$ Reference Level: $<65$ & 1.04 & $0.126 ; 8.478$ \\
\hline $\begin{array}{l}\text { Previous Fracture After } 50 \text { y } \\
\text { *Reference Level: no fracture after } 50\end{array}$ & 2.16 & $1.201 ; 3.874$ \\
\hline $\begin{array}{l}\text { Maternal History of Fracture } \\
\text { *Reference Level: no family history }\end{array}$ & 2.89 & $1.035 ; 8.081$ \\
\hline $\begin{array}{l}\text { Osteoporotic (<-2.5 SD)** } \\
\text { *Reference Level: normal BMD }\end{array}$ & 3.89 & $0.367 ; 41.263$ \\
\hline
\end{tabular}

efficient measure of gait speed had the same discriminant value for fracture prediction as femoral BMD at all cutoff values [36]. It was the prospective study by Cummings et al. [10] that indicated the importance of examining one's ability to rise from a chair without the use of arms in risk assessment. They determined that this factor alone was the most significant at predicting hip fracture and that the addition of other neuromuscular assessment tests added little to the prediction of subsequent hip fracture [10].

The use of arms to rise from a seated position was found to be the single most important predictor of increased fracture risk at the hip in CANDOO patients. These results indicate that both research and clinical settings should place a greater emphasis than is currently standard procedure on this variable or others that examine neuromuscular ability. Such an easily assessed risk factor would make for an efficient and effective mechanism to gauge risk across the entire population, especially in those for which BMD testing is not feasible.

Many of these risk factors have been successfully incorporated into clinical risk assessment tools, while others such as the use of arms to rise from a chair tend to be ignored. A variety of current risk assessment mechanisms are used routinely in clinical practice, including the Osteoporosis Risk Assessment Instrument (ORAI), Simple Calculated Osteoporosis Risk Estimation (SCORE) and the module Physicians' Information and Education Resource (PIER) [37-39].

The ORAI is a clinical assessment tool that was designed to identify women over the age of 45 who are at increased risk for osteoporosis who should undergo BMD testing. This simple tool uses three items (age, weight and estro- gen use) to gauge risk and has been validated to identify over $90 \%$ of women at increased risk of osteoporotic fracture while ensuring that less than $50 \%$ of those with normal BMD are selected [37]. Although this procedure has proven to be useful in clinically identifying patients at risk, it only allows for examination of females and relies completely on historical risk factors.

A similar mechanism for identifying individuals at increased risk in clinical practice was set forth in SCORE. This tool relies on six risk factors rather than three in order to assess risk of osteoporotic fracture. These six variables include: race, age, rheumatoid arthritis, history of nontraumatic fracture after age 45, weight and estrogen use [37]. Despite its incorporation of more risk factors, SCORE has been shown to have similar sensitivity to ORAI but greater selection of individuals with normal BMD [40].

The effectiveness of determining those individuals at increased risk of future fracture not only revolves around public awareness of risk factors, but relies heavily on the ability of physicians (especially in primary care settings) to effectively and efficiently identify at-risk individuals. Module PIER [39] is a web and computer based resource designed to guide physicians through the diagnosis, treatment and management of a plethora of diseases including osteoporosis. Their recommendations include the use of the Fracture Index for risk assessment and analyses of other variables including use of corticosteroid therapy for more than 3 months, impaired vision, low calcium intake, low physical activity, dementia, alcohol consumption of greater than 2 drinks per day and estrogen deficiency before 45 years of age. Subsequently, any post-menopausal woman over 65 years of age or those under 65 who 
have at least one of these risk factors is recommended to have her BMD assessed. The inclusion of risk factors to assess frailty and mobility in PIER, such as the use of arms to rise from a chair and impaired vision, takes into consideration the importance of preventing falls and future fracture rather than solely basing risk assessment on historical risk factors.

Our study incorporated a large sample of post-menopausal women from the CANDOO database. The subject data from this database were homogeneous as they stemmed from a group of patients who were assessed in the tertiary care setting and represented a "real world" group [13]. Moreover, the multivariable analyses involved detailed and controlled delineation of potential confounding variables.

Although the study design attempted to minimize limiting factors, it should be recognized that much of the information in the CANDOO database relied on factual recall from patients, some of which was reviewed at considerable length after the experience had occurred. In addition, although careful delineation of confounding variables was carried out, it remains uniquely possible that other potential confounders played a role. Assessment of fractures for the CANDOO database were either confirmed or self-reported and as a result sub-clinical fractures in patients could have been missed. Also, the lack of data on male patients meant only females were assessed and, as such, results should not necessarily be extrapolated to the male population.

Further studies will need to be undertaken in attempt to identify a more definitive and indicative group of risk factors that can be used across genders and populations to assess future fracture risk and the subsequent development of osteoporosis.

\section{Conclusion}

Despite extensive research and new treatment strategies, osteoporosis remains one of the "sleeping giants of health care". Much of the population remains undiagnosed and unaware of the importance of early recognition and preventative treatment. As the "boomer bulge" continues to progress through middle age and into senior brackets, the effects of osteoporosis on both the individual and health care system will be enormous. This study has shown that there exists a variety of predictors of future fracture, besides $\mathrm{BMD}$, that can be easily assessed by a physician. The predictive significance of each of these risk factors has been shown to be dependent on the site of incident fracture. The assessment of unconventional risk factors such as examining one's ability to rise from a chair without the use of arms to gauge proprioception, strength and coordination are simple, convenient and valuable in terms of predicting fracture risk. These risk assessment factors can be easily incorporated into routine clinical practice, especially where BMD testing is unfeasible. A better understanding of which factors lead to an increase in the incidence of fractures is a crucial step in evaluating patients at risk and designing therapeutic strategies.

\section{Competing interests}

The author(s) declare that they have no competing interests.

\section{Authors' contributions}

RB- conceived design, participated in analysis and coordination and drafted manuscript

JDA- conceived design, participated in CANDOO database, participated in analysis and manuscript

AP- conceived design, participated in CANDOO database, coordinated analysis and participated in manuscript

GI- performed statistical analysis and participated in study design

WO- participated in CANDOO, study design, and analysis

RS- participated in CANDOO, study design, and analysis

TM- participated in CANDOO, study design, and analysis

RJ- participated in CANDOO, study design, and analysis

JB- participated in CANDOO, study design, and analysis

DH- participated in CANDOO, study design, and analysis

AP- participated in CANDOO, study design, and analysis

MP- participated in CANDOO, study design, and analysis

CG- participated in statistical analysis and study design

\section{Acknowledgements}

McMaster University is the institution to which this work should be attributed.

\section{References}

I. Brown JP, Josse RG, Scientific Advisory Council of the Osteoporosis Society of Canada: 2002 clinical practice guidelines for the diagnosis and management of osteoporosis in Canada. CMA 2003:I-34.

2. Matthis C, Weber U, O'Neill TW, Raspe H: Health impact associated with vertebral deformities: results from the European Vertebral Osteoporosis Study (EVOS). Osteoporos Int 1998, 8:364-372.

3. Melton LJIII, Chrischilles EA, Cooper C, Lane AW, Riggs BL: Perspective. How many women have osteoporosis? J Bone Miner Res 1992, 7:1005-1010. 
4. Goeree ROB, Pettitt DB, Cuddy L, Ferraz M, Adachi J: An assessment of the burden of illness due to osteoporosis in Canada. J Soc Obstet Gynaecol Can 1996, I 8: I 5-24.

5. Wiktorowicz ME, Goeree R, Papaioannou A, Adachi JD, Papadimitropoulos E: Economic implications of hip fracture: health service use, institutional care and cost in Canada. Osteoporos Int 200I, I 2:27|-278.

6. Schott AM, Cormier C, Hans D, Favier F, Hausherr E, Dargent-Molina P, Delmas PD, Ribot C, Sebert JL, Breart G, Meunier PJ: How hip and whole-body bone mineral density predict hip fracture in elderly women: The EPIDOS prospective study. Osteoporosis International 1998, 8:247-254.

7. Marshall $\mathrm{D}$, Johnell $\mathrm{O}$, Wedel $\mathrm{H}$ : Meta-analysis of how well measures of bone mineral density predict occurrence of osteoporotic fractures. British Medical Journal | 996, 3 | 2: | 254- I 259.

8. Genant HK, Li J, Wu CY, Shepherd JA: Vertebral fractures in osteoporosis - A new method for clinical assessment. Journal of Clinical Densitometry 2000, 3:28I-290.

9. Nelson HD, Helfand M, Woolf SH, Allan JD: Screening for postmenopausal osteoporosis: A review of the evidence for the US Preventive Services Task Force. Annals of Internal Medicine 2002, I 37:529-54I.

10. Cummings SR, Nevitt MC, Browner WS: Risk factors for hip fracture in white women. The New England Journal of Medicine 1995, 332:767-773.

II. Torgerson DJ, Campbell MK, Thomas RE, Reid DM: Prediction of perimenopausal fractures by bone mineral density and other risk factors. J Bone Mine Res 1996, I I:293-297.

12. D.M B, M S, L P, P DM, R L, M.S H, O J: An Assessment Tool for Predicting Fracture Risk in Postmenopausal Women. Osteoporosis International 200I, I 2:519-528.

13. Olszynski WP, loannidis G, Sebaldt RJ, Hanley DA, Petrie A, Brown JP, Josse RG, Murray TM, Goldsmith CH, Stephenson GF, Papaioannou A, Adachi JD: The association between iliocostal distance and the number of vertebral and non-vertebral fractures in women and men registered in the Canada Database of Osteoporosis and Osteopenia (CANDOO). BMC Musculoskelet Disord 2002, 3:22 [http://www.biomedcentral.com/|47|-2474/3/22].

14. Rubin D: Inference and missing data. Biometrika 1976 , 63:58I-590.

15. Schafer JL: Analysis of Incomplete Multivariate Data London, UK, Chapman \& Hall; 1997:2-6.

16. McClung MR: Therapy for fracture prevention. JAMA 1999, 282:687-689.

17. Kanis JA, Delmas P, Burckhardt P, Cooper C, Torgerson D: Guidelines for diagnosis and management of osteoporosis. The European Foundation for Osteoporosis and Bone Disease. Osteoporos Int 1997, 7:390-406.

18. Kanis JA, O J, Oden A, Dawson A, C DL, Jonsson B: Ten Year Probabilities of Osteoporotic Fractures According to BMD and Diagnostic Thresholds. Osteoporosis International 2001, 1 2:989-995.

19. Cummings SR, Black DM, Nevitt MC, Browner W, Cauley J, Ensrud K, Genant HK, Palermo L, Scott J, Vogt TM: Bone density at various sites for prediction of hip fractures. The Study of Osteoporotic Fractures Research Group. Lancet 1993, 341:72-75.

20. Wasnich RD, Davis JW, Ross PD: Spine fracture risk is predicted by non-spine fractures. Osteoporos Int 1994, 4: I-5.

21. Davis JW, Grove JS, Wasnich RD, Ross PD: Spatial relationships between prevalent and incident spine fractures. Bone 1999, 24:26I-264

22. A.A I, W C, C C, J.D F: Prevalent Vertebral Deformity Predicts Incident Hip though not distal Forearm Fracture: Results from the European Prospective Osteoporsis Study. Osteoporosis International 200 I, I 2:85-90.

23. Klotzbuecher CM, Ross PD, Landsman PB, Abbott TAIII, Berger M: Patients with prior fractures have an increased risk of future fractures: a summary of the literature and statistical synthesis. J Bone Miner Res 2000, I 5:721-739.

24. Ross PD, Davis JW, Epstein RS, R.D W: Pre-existing fractures and bone mass predict vertebral fracture incidence in women. Annals of Internal Medicine 199I, I | 4:919-923.

25. Tromp AM, Smit JH, Deeg DJ, Bouter LM, Lips P: Predictors for falls and fractures in the Longitudinal Aging Study Amsterdam. J Bone Miner Res 1998, 13:1932-1939.
26. Black DM, Palermo L, Nevitt MC, Genant HK, Christensen L, Cummings SR: Defining incident vertebral deformity: a prospective comparison of several approaches. The Study of Osteoporotic Fractures Research Group. J Bone Miner Res 1999, I4:90-101.

27. Fox KM, Cummings SR, E W, K S: Femoral Neck and Intertrochanteric Fractures Have Different Risk Factors: A Prospective Study. Osteoporosis International 2000, I I : I0 I8-1023.

28. Ettinger B, Black DM, Mitlak BH, Knickerbocker RK, Nickelsen T, Genant HK, Christiansen C, Delmas PD, Zanchetta JR, Stakkestad J, Gluer CC, Krueger K, Cohen FJ, Eckert S, Ensrud KE, Avioli LV, Lips $P$, Cummings SR: Reduction of vertebral fracture risk in postmenopausal women with osteoporosis treated with raloxifene: results from a 3-year randomized clinical trial. Multiple Outcomes of Raloxifene Evaluation (MORE) Investigators. JAMA 1999, 282:637-645.

29. Black DM, Arden NK, Palermo L, Pearson J, Cummings SR: Prevalent vertebral deformities predict hip fractures and new vertebral deformities but not wrist fractures. Study of Osteoporotic Fractures Research Group. J Bone Miner Res 1999, | 4:821-828.

30. Cooper C, Atkinson EJ, O'Fallon WM, Melton LJIII: Incidence of clinically diagnosed vertebral fractures: a population-based study in Rochester, Minnesota, 1985-1989. J Bone Miner Res 1992, 7:221-227.

3I. Honkanen R, Tuppurainen M, Kroger H, Alhava E, Saarikoski S: Relationships between risk factors and fractures differ by type of fracture: a population-based study of $I 2,192$ perimenopausal women. Osteoporos Int 1998, 8:25-31.

32. Siris ES, Miller PD, Barrett-Connor E, Faulkner KG, Wehren LE, Abbott TA, Berger ML, Santora AC, Sherwood LM: Identification and fracture outcomes of undiagnosed low bone mineral density in postmenopausal women: results from the National Osteoporosis Risk Assessment. JAMA 200I, 286:28I5-2822

33. Law MR, Hackshaw AK: A meta-analysis of cigarette smoking, bone mineral density and risk of hip fracture: recognition of a major effect. BMJ 1997, 3 I 5:84I-846.

34. Law MR, Cheng R, Hackshaw AK, Allaway S, Hale AK: Cigarette smoking, sex hormones and bone density in women. Eur J Epidemiol 1997, I 3:553-558.

35. Dargent-Molina P, Favier F, Grandjean H, Baudoin C, Schott AM, Hausherr E, Meunier PJ, Breart G: Fall-related factors and risk of hip fracture: the EPIDOS prospective study. Lancet 1996, 348: $145-149$

36. Dargent-Molina P, Schott AM, Hans D, Favier F, Grandjean H, Baudoin C, Meunier PJ, Breart G: Separate and combined value of bone mass and gait speed measurements in screening for hip fracture risk: results from the EPIDOS study. Epidemiologie de l'Osteoporose. Osteoporos Int 1999, 9:188-192.

37. Cadarette SM, Jaglal SB, Kreiger N, Mclsaac WJ, Darlington GA, Tu JV: Development and validation of the Osteoporosis Risk Assessment Instrument to facilitate selection of women for bone densitometry. CMA/ 2000, I 62: I289-I294.

38. Cadarette SM, Jaglal SB, Murray TM, Mclsaac WJ, Joseph L, Brown JP: Evaluation of decision rules for referring women for bone densitometry by dual-energy $X$-ray absorptiometry. Journal of the American Medical Association 2001, 286:57-63.

39. Papaioannou A, Ferko N, Adachi JD, Watts N: Physicians' Information and Education Resource (PIER). 2004 [http://pier.acpon line.org/index.html].

40. Cadarette SM, Jaglal SB, Murray TM: Validation of the simple calculated osteoporosis risk estimation (SCORE) for patient selection for bone densitometry. Osteoporos Int 1999, 10:85-90.

\section{Pre-publication history}

The pre-publication history for this paper can be accessed here:

http://www.biomedcentral.com/1471-2474/6/47/prepub 